\title{
The thermodynamic Casimir effect in the neighbourhood of the $\lambda$-transition: A Monte Carlo study of an improved three dimensional lattice model
}

\author{
Martin Hasenbusch \\ Institut für Physik, Humboldt-Universität zu Berlin \\ Newtonstr. 15, 12489 Berlin, Germany \\ e-mail: $\quad$ Martin.Hasenbusch@physik.hu-berlin.de
}

\begin{abstract}
We study the thermodynamic Casimir effect in thin films in the three dimensional XY universality class. To this end, we simulate the improved two component $\phi^{4}$ model on the simple cubic lattice. We use lattices up to the thickness $L_{0}=33$. Based on the results of our Monte Carlo simulations we compute the universal finite size scaling function $\theta$ that characterizes the behaviour of the thermodynamic Casimir force in the neighbourhood of the critical point. We confirm that leading corrections to the universal finite size scaling behaviour due to free boundary conditions can be expressed by an effective thickness $L_{0, \text { eff }}=L_{0}+L_{s}$, with $L_{s}=1.02(7)$. Our results are compared with experiments on films of ${ }^{4} \mathrm{He}$ near the $\lambda$-transition, previous Monte Carlo simulations of the XY model on the simple cubic lattice and fieldtheoretic results. Our result for the finite size scaling function $\theta$ is essentially consistent with the experiments on films of ${ }^{4} \mathrm{He}$ and the previous Monte Carlo simulations.
\end{abstract}

Keywords: $\lambda$-transition, Classical Monte Carlo simulation, thin films, thermodynamic Casimir effect 


\section{Introduction}

In 1978 Fisher and de Gennes [1] realized that there should be a so called "thermodynamic" Casimir effect. This means that a force emerges when thermal fluctuations are restricted by a container. Thermal fluctuations extend to large scales in the neighbourhood of critical points. In the thermodynamic limit, in the neighbourhood of the critical point, various quantities diverge following power laws. E.g. the correlation length, which measures the spatial extension of fluctuations, behaves as

$$
\xi \simeq \xi_{0, \pm} t^{-\nu}
$$

where $t=\left(T-T_{c}\right) / T_{c}$ is the reduced temperature and $T_{c}$ the critical temperature. $\xi_{0,+}$ and $\xi_{0,-}$ are the amplitude of the correlation length in the high and low temperature phase, respectively. While $\xi_{0,+}$ and $\xi_{0,-}$ depend on the microscopic details of the system, the critical exponent $\nu$ and the ratio $\xi_{0,+} / \xi_{0,-}$ are universal. This means that they assume exactly the same values for all systems within a given universality class. A universality class is characterized by the spatial dimension of the system, the range of the interaction and the symmetry of the order parameter. The modern theory of critical phenomena is the Renormalization Group (RG). For reviews see e.g. [2, 3, 4, 5]. Here we consider the XY universality class in three dimensions with short range interactions. This universality class is of particular interest, since the $\lambda$-transition of ${ }^{4} \mathrm{He}$ is supposed to share this universality class. The most accurate experimental results for critical exponents and universal amplitude ratios for a three dimensional system have been obtained for this transition; for a review see [6].

The critical behaviour is modified by a confining geometry. If the system is finite in all directions, thermodynamic functions have to be analytic. I.e. a singular behaviour like eq. (11) is excluded. As a remnant of such singularities there remains a peak in the neighbourhood of the transition. With increasing linear extension the hight of the peak increases and the temperature of the maximum approaches the critical temperature. This behaviour is described by the theory of finite size scaling (FSS). For reviews see [7, 8]. In general the physics in the neighbourhood of the transition is governed by the ratio $L_{0} / \xi$, where $L_{0}$ is the linear extension of the container and $\xi$ the correlation length of the bulk system. Furthermore it depends on the geometry of the container and on the type of the boundary conditions that the container imposes on the order parameter. For a review on experimental studies of ${ }^{4}$ He near the $\lambda$-transition in confining geometries see [9].

Here we study thin films. Thin films are finite in one direction and infinite in the other two directions. In this case singular behaviour is still possible. However the associated phase transition belongs to the two-dimensional universality class. 
I.e. in the case of $U(1)$ symmetry, a Kosterlitz-Thouless (KT) transition [10, 11, 12] is expected. In [13] we have confirmed the KT-nature of this transition and have studied the scaling of the transition temperature with the thickness of the film. Recently [14] we determined the finite size scaling behaviour of the specific heat of thin films. Here we investigate the thermodynamic Casimir force in thin films in the three dimensional XY universality class.

From a thermodynamic point of view, the Casimir force per unit area is given by

$$
F_{\text {casimir }}=-\frac{\partial \tilde{f}_{e x}}{\partial L_{0}}
$$

where $L_{0}$ is the thickness of the film and $\tilde{f}_{e x}=\tilde{f}_{f i l m}-L_{0} \tilde{f}_{3 D}$ is the excess free energy per area of the film, where $\tilde{f}_{\text {film }}$ is the free energy per area of the film and $\tilde{f}_{3 D}$ the free energy density of the thermodynamic limit of the three dimensional system; see e.g. [15]. Finite size scaling predicts that the Casimir force behaves as

$$
F_{\text {casimir }}=\frac{k_{B} T}{L_{0}^{3}} \theta\left(t\left[L_{0} / \xi_{0}\right]^{1 / \nu}\right)
$$

where $\theta(x)$ is a universal finite size scaling function. 11 In [16, 17] ${ }^{4} \mathrm{He}$ films of thicknesses up to $588 \AA$ have been studied. These experiments show clearly that the thermodynamic Casimir force is indeed present. Throughout it is negative. In the low temperature phase of the three dimensional bulk system it shows a pronounced minimum. The data are essentially consistent with the prediction eq. (3)). The minimum of $\theta(x)$ is located at $x=t\left(L_{0} / \xi_{0}\right)^{1 / \nu} \approx-5.5$.

It has been a challenge for theorists to compute the finite size scaling function $\theta(x)$. Krech and Dietrich [18, 19] have computed it in the high temperature phase using the $\epsilon$-expansion up to $\mathrm{O}(\epsilon)$. This result is indeed consistent with the measurements on ${ }^{4} \mathrm{He}$ films. Deep in the low temperature phase, the spin wave approximation should provide an exact result. It predicts a negative non-vanishing value for $\theta(x)$. However the experiments suggest a much larger absolute value for $\theta(x)$ in this region. Until recently a reliable theoretical prediction for the minimum of $\theta(x)$ and its neighbourhood was missing. Using a renormalized mean-field approach the authors of [20] have computed $\theta(x)$ for the whole temperature range. Qualitatively they reproduce the features of the experimental result. However the position of the minimum is by almost a factor of 2 different from the experimental one. The value at the minimum is wrongly estimated by a factor of about 5 .

\footnotetext{
${ }^{1}$ Following the literature, in eq. (3i) we shall use $\xi_{0,+}$ in the following.
} 
Only quite recently Monte Carlo simulations of the XY model on the simple cubic lattice [21, 22, 23] provided results for $\theta(x)$ which essentially reproduce the experiments on ${ }^{4} \mathrm{He}$ films [16, 17]. These simulations were performed with lattices of a thickness up to $L_{0}=16$ [22] and up to $L_{0}=20$ [23]. The authors of [23] pointed out that for these lattice sizes corrections to scaling still play an important role. The purpose of the present work is to get accurate control over the leading corrections to scaling, allowing us to compute $\theta(x)$ with reliable error bars.

As the first step in this direction we simulate the improved two-component $\phi^{4}$ model instead of the XY model. For a precise definition of these models see the section below. This way we avoid leading corrections to scaling which are $\propto L_{0}^{-\omega}$ with $\omega=0.785(20)$ [24]; similar values for the exponent are obtained with field-theoretic methods; for a review see e.g. [5]. In order to mimic the vanishing order parameter that is observed at the boundaries of ${ }^{4} \mathrm{He}$ films, Dirichlet boundary conditions with vanishing field are imposed. These lead to corrections $\propto L_{0}^{-1}$ [25]. These corrections can be eliminated by replacing the thickness $L_{0}$ by an effective one $L_{0, \text { eff }}=L_{0}+L_{s}$, where $L_{s}=1.02(7)$ [13] for the model that we have simulated. 2

This paper is organized as follows: First we define the model and the observables that we have measured. Next we discuss the finite size scaling behaviour of the Casimir force. In particular, we discuss corrections to scaling caused by the Dirichlet boundary conditions. We outline the method used to compute the Casimir force. We discuss the simulations that have been performed and analyze our data. We compare our results with experiments [16, 17], previous Monte Carlo simulations [22, 23] and the $\epsilon$-expansion [18, 19]. Finally we summarize and conclude.

\section{The model and the observables}

We study the two component $\phi^{4}$ model on the simple cubic lattice. We label the sites of the lattice by $x=\left(x_{0}, x_{1}, x_{2}\right)$. The components of $x$ might assume the values $x_{i} \in\left\{1,2, \ldots, L_{i}\right\}$. We simulate lattices of the size $L_{1}=L_{2}=L$ and $L_{0} \ll L$. In 1 and 2-direction we employ periodic boundary conditions and free boundary conditions in 0 -direction. This means that the sites with $x_{0}=1$ and $x_{0}=L_{0}$ have only five nearest neighbours. This type of boundary conditions could be interpreted as Dirichlet boundary conditions with 0 as value of the field at $x_{0}=0$

\footnotetext{
${ }^{2}$ In the literature, replacing $L_{0}$ by $L_{0, \text { eff }}=L_{0}+L_{s}$ to account for surface corrections, was first discussed by Capehart and Fisher [26] in the context of the surface susceptibility of Ising films.
} 
and $x_{0}=L_{0}+1$. Note that viewed this way, the thickness of the film is $L_{0}+1$ rather than $L_{0}$. This provides a natural explanation of the result $L_{s}=1.02(7)$ obtained in [13] and might be a good starting point for a field theoretic calculation of $L_{s}$. The Hamiltonian of the two component $\phi^{4}$ model, for a vanishing external field, is given by

$$
\mathcal{H}=-\beta \sum_{<x, y>} \vec{\phi}_{x} \cdot \vec{\phi}_{y}+\sum_{x}\left[\vec{\phi}_{x}^{2}+\lambda\left(\vec{\phi}_{x}^{2}-1\right)^{2}\right]
$$

where the field variable $\vec{\phi}_{x}$ is a vector with two real components. $\langle x, y\rangle$ denotes a pair of nearest neighbour sites on the lattice. The partition function is given by

$$
Z=\prod_{x}\left[\int d \phi_{x}^{(1)} \int d \phi_{x}^{(2)}\right] \exp (-\mathcal{H}) .
$$

Note that following the conventions of our previous work, e.g. 28, we have absorbed the inverse temperature $\beta$ into the Hamiltonian. 3 In the limit $\lambda \rightarrow \infty$ the field variables are fixed to unit length; i.e. the XY model is recovered. For $\lambda=0$ we get the exactly solvable Gaussian model. For $0<\lambda \leq \infty$ the model undergoes a second order phase transition that belongs to the XY universality class. Numerically, using Monte Carlo simulations and high-temperature series expansions, it has been shown that there is a value $\lambda^{*}>0$, where leading corrections to scaling vanish. Numerical estimates of $\lambda^{*}$ given in the literature are $\lambda^{*}=2.10(6)$ [27], $\lambda^{*}=2.07(5)$ [28] and most recently $\lambda^{*}=2.15(5)$ [24]. The inverse of the critical temperature $\beta_{c}$ has been determined accurately for several values of $\lambda$ using finite size scaling (FSS) 24]. We shall perform our simulations at $\lambda=2.1$, since for this value of $\lambda$ comprehensive Monte Carlo studies of the three-dimensional system in the low and the high temperature phase have been performed [13, 24, 29, 30. At $\lambda=2.1$ one gets $\beta_{c}=0.5091503(6)$ [24]. Since $\lambda=2.1$ is not exactly equal to $\lambda^{*}$, there are still corrections $\propto L^{-\omega}$, although with a small amplitude. In fact, following [24], it should be by at least a factor 20 smaller than for the standard XY model.

In [13] we find for $\lambda=2.1$ by fitting the data for the second moment correlation length in the high temperature phase

$$
\xi_{2 n d}=0.26362(8) t^{-0.6717} \times\left[1+0.039(8) t^{0.527}-0.72(4) t\right],
$$

where $t=0.5091503-\beta$. Here we shall use $\nu=0.6717(1)$ 24 as value of the critical exponent of the correlation length. Recent experiments on the $\lambda$-transition of ${ }^{4} \mathrm{He}$

\footnotetext{
${ }^{3}$ Therefore, following [4] we actually should call it reduced Hamiltonian.
} 
suggest a slightly smaller value: $\nu=0.6709(1)$ [31]. This discrepancy is however not crucial for the present study. Note that in the high temperature phase there is little difference between $\xi_{2 n d}$ and the exponential correlation length $\xi_{\text {exp }}$ which is defined by the asymptotic decay of the two-point correlation function. Following [28]:

$$
\lim _{t \rightarrow 0} \frac{\xi_{\text {exp }}}{\xi_{2 n d}}=1.000204(3), \quad(t>0)
$$

for the thermodynamic limit of the three-dimensional system.

\subsection{The internal energy and the free energy}

The reduced free energy density is defined as

$$
f=-\frac{1}{L_{0} L_{1} L_{2}} \log Z
$$

I.e. compared with the free energy density $\tilde{f}$, a factor $k_{B} T$ is skipped.

Note that in eq. (4) $\beta$ does not multiply the second term. Therefore, strictly speaking, $\beta$ is not the inverse of $k_{B} T$. In order to study universal quantities it is not crucial how the transition line in the $\beta-\lambda$ plane is crossed, as long as this path is not tangent to the transition line. Therefore, following computational convenience, we vary $\beta$ at fixed $\lambda$. Correspondingly we define the (internal) energy density as the derivative of the reduced free energy density with respect to $\beta$. Furthermore, to be consistent with our previous work [14], we multiply by -1 :

$$
E=\frac{1}{L_{0} L_{1} L_{2}} \frac{\partial \log Z}{\partial \beta}
$$

It follows

$$
E=\frac{1}{L_{0} L_{1} L_{2}}\left\langle\sum_{<x, y>} \vec{\phi}_{x} \cdot \vec{\phi}_{y}\right\rangle,
$$

which can be easily determined in Monte Carlo simulations. From eqs. (8],9) it follows that the free energy density can be computed as

$$
f(\beta)=f\left(\beta_{0}\right)-\int_{\beta_{0}}^{\beta} \mathrm{d} \tilde{\beta} E(\tilde{\beta}) .
$$




\section{The finite size scaling behaviour of the thermal Casimir force}

Let us discuss the scaling behaviour of the reduced excess free energy. Since we study an improved model we ignore corrections $\propto L_{0}^{-\omega}$ in the following. We take into account leading corrections due to the boundary conditions by replacing the thickness $L_{0}$ of the film by $L_{0, e f f}=L_{0}+L_{s}$ at the appropriate places. We split the free energies in singular (s) and non-singular (ns) parts:

$$
\begin{aligned}
f_{\text {ex }}\left(t, L_{0}\right) & =f_{\text {film }}\left(t, L_{0}\right)-L_{0} f_{3 D}(t) \\
& =f_{f i l m, s}\left(t, L_{0}\right)+L_{0, e f f, n s} f_{n s}(t)-L_{0} f_{3 D, s}(t)-L_{0} f_{n s}(t) \\
& =L_{0, e f f}^{-2} h(x)+L_{s} f_{3 D, s}(t)+L_{n s} f_{n s}(t)
\end{aligned}
$$

where $h(x)=L_{0, e f f}^{2}\left[f_{f i l m, s}\left(t, L_{0}\right)-L_{0, e f f} f_{3 D, s}(t)\right]$ is a universal finite size scaling function and $x=t\left[L_{0, e f f} / \xi_{0}\right]^{1 / \nu}$. Following RG theory the non-singular part is not affected by finite size effects. However it is not clear a priori how Dirichlet boundary conditions affect the non-singular part of the free energy. Therefore we allow for

$L_{n s}=L_{0, e f f, n s}-L_{0} \neq 0$ and $L_{n s} \neq L_{s}$. Taking the derivative with respect to $L_{0}$ we get the thermodynamic Casimir force per area [15]

$$
\beta F_{\text {casimir }}=-\frac{\partial f_{e x}\left(t, L_{0}\right)}{\partial L_{0}}=2 L_{0, e f f}^{-3} h(x)-L_{0, e f f}^{-3} \frac{1}{\nu} x h^{\prime}(x)=L_{0, e f f}^{-3} \theta(x)
$$

where $\theta(x)=2 h(x)-\frac{1}{\nu} x h^{\prime}(x)$.

\section{Computing the Casimir force on the lattice}

Here we follow essentially the approach of [22]. For an alternative method see [21, 23]. On the lattice the thickness $L_{0}$ assumes integer values. Therefore we approximate the derivative for half-integer values of $L_{0}$ as

$$
\left.\frac{\partial f_{e x}(\beta, L)}{\partial L}\right|_{L=L_{0}} \approx \Delta f_{e x}\left(\beta, L_{0}\right)=f\left(\beta, L_{0}+1 / 2\right)-f\left(\beta, L_{0}-1 / 2\right)+f_{3 D}(\beta) .
$$

Correspondingly we define

$$
\Delta E_{e x}\left(\beta, L_{0}\right)=E\left(\beta, L_{0}+1 / 2\right)-E\left(\beta, L_{0}-1 / 2\right)-E_{3 D}(\beta)
$$


where $E\left(\beta, L_{0}\right)$ is the energy per area of a thin film and $E_{3 D}(\beta)$ the energy density of the three dimensional system. Analogous to eq. (11) we compute

$$
\Delta f_{e x}(\beta)=-\int_{\beta_{0}}^{\beta} \mathrm{d} \tilde{\beta} \Delta E_{e x}(\tilde{\beta})
$$

where $\beta_{0}$ is chosen such that $\xi\left(\beta_{0}\right) \ll L_{0}$ and hence the Casimir force vanishes.

\section{$5 \quad$ Numerical results}

In 14 we have studied the specific heat of thin films in the two component $\phi^{4}$ model at $\lambda=2.1$. To this end, we had determined the energy density for the three dimensional thermodynamic limit and for films of the thicknesses $L_{0}=8,16$ and 32 for a large number of $\beta$-values. In order to compute the derivative with respect to $L_{0}$, we have complemented these simulations by ones for $L_{0}=9,17$ and 33. The Monte Carlo algorithm that has been used is the same as in [14]: An update-cycle is composed of a Metropolis sweep, a few overrelaxation sweeps and single [32 and wall [33] cluster updates. One sweep means that a local update is performed at each site of the lattice ones. As random number generator we have used the SIMDoriented Fast Mersenne Twister algorithm [34]. In table 1] we have summarized the statistics of our runs. In total these simulations took about 3 years of CPU-time on a single core of a Quad-Core Opteron(tm) $2378 \mathrm{CPU}(2.4 \mathrm{GHz})$.

Using these data, we have computed $\Delta E_{e x}$ for $L_{0}=8.5,16.5$ and 32.5. One should note that the statistical error of $E\left(\beta, L_{0}+1 / 2\right)-E\left(\beta, L_{0}-1 / 2\right)$ is much larger than that of $E_{3 D}(\beta)$. In order to obtain $\Delta f_{e x}$ we have numerically integrated $\Delta E_{e x}$ using the trapezoidal rule:

$$
-\Delta f_{e x}\left(\beta_{n}\right) \approx \sum_{i=0}^{n-1} \frac{1}{2}\left(\beta_{i+1}-\beta_{i}\right)\left(\Delta E_{e x}\left(\beta_{i+1}\right)+\Delta E_{e x}\left(\beta_{i}\right)\right)
$$

where $\beta_{i}$ are the values of $\beta$ we have simulated at. They are ordered such that $\beta_{i+1}>\beta_{i}$ for all $i$. We have chosen $\beta_{0}=0.49,0.5$ and 0.505 for $L_{0}=8.5,16.5$ and 32.5. We find that $\Delta E_{\text {ex }}$ is equal to zero within error bars up to values of $\beta$ that are slightly larger than the $\beta_{0}$ that we have chosen.

The estimate obtained from the integration is affected by statistical and systematical errors. The statistical one can be easily computed, since the $\Delta E_{e x}$ are obtained from independent simulations:

$$
\epsilon^{2}\left(-\Delta f_{e x}\left(\beta_{n}\right)\right)=\frac{\left(\beta_{1}-\beta_{0}\right)^{2}}{4} \epsilon^{2}\left[\Delta E_{e x}\left(\beta_{0}\right)\right]+\frac{\left(\beta_{n}-\beta_{n-1}\right)^{2}}{4} \epsilon^{2}\left[\Delta E_{e x}\left(\beta_{n}\right)\right]
$$


Table 1: We characterize our new simulations. In the first column we give the thickness $L_{0}$ of the film. In the second column we give the linear size $L=L_{1}=L_{2}$ of the lattice in the other two directions. In the third and fourth column we give the upper and lower bound of the interval in $\beta$ that has been simulated. In the fifth column we give the step size $\Delta \beta$ that we used. E.g. $\beta_{\min }=0.49, \beta_{\max }=0.519$ and $\Delta \beta=0.001$ means that $\beta=0.49,0.491,0.492, \ldots, 0.519$ have been simulated. Finally, in the last column we give the number of measurements (stat) that we have performed for each of the simulations.

\begin{tabular}{|c|r|l|l|l|c|}
\hline$L_{0}$ & $L_{1}=L_{2}$ & $\beta_{\min }$ & $\beta_{\max }$ & $\Delta \beta$ & stat \\
\hline 9 & 64 & 0.49 & 0.519 & 0.001 & $5 \times 10^{5}$ \\
9 & 128 & 0.52 & 0.527 & 0.001 & $2 \times 10^{5}$ \\
9 & 256 & 0.528 & 0.56 & 0.001 & $10^{5}$ \\
9 & 256 & 0.562 & 0.58 & 0.002 & $10^{5}$ \\
9 & 512 & 0.536 & 0.539 & 0.001 & $10^{5}$ \\
9 & 512 & 0.5395 & 0.548 & 0.0005 & $10^{5}$ \\
9 & 512 & 0.548 & 0.57 & 0.001 & $10^{5}$ \\
9 & 1024 & 0.539 & 0.548 & 0.0005 & $10^{5}$ \\
\hline 17 & 256 & 0.527 & 0.55 & 0.001 & $2 \times 10^{5}$ \\
17 & 512 & 0.5 & 0.512 & 0.001 & $10^{5}$ \\
17 & 512 & 0.5125 & 0.529 & 0.0005 & $10^{5}$ \\
17 & 512 & 0.53 & 0.55 & 0.001 & $10^{5}$ \\
17 & 1024 & 0.5205 & 0.529 & 0.0005 & $8 \times 10^{4}$ \\
\hline 33 & 256 & 0.502 & 0.50875 & 0.00025 & $4 \times 10^{5}$ \\
33 & 512 & 0.509 & 0.5128 & 0.0002 & $3 \times 10^{5}$ \\
33 & 1024 & 0.513 & & & $10^{5}$ \\
33 & 1024 & 0.5132 & & & $8 \times 10^{4}$ \\
\hline
\end{tabular}




$$
+\sum_{i=1} n-1 \frac{\left(\beta_{i+1}-\beta_{i-1}\right)^{2}}{4} \epsilon^{2}\left[\Delta E_{e x}\left(\beta_{i}\right)\right]
$$

where $\epsilon^{2}$ denotes the square of the statistical error.

In order to estimate the error due to the finite step size $\beta_{i+1}-\beta_{i}$ we have redone the integration, skipping every second value of $\beta$; i.e. doubling the step size. In all three cases (i.e. $L_{0}=8.5,16.5$ and 32.5 ), the results were consistent within the statistical errors. Therefore we are confident that the systematical error due to the finite step size is smaller than the statistical one.

In figure1 we have plotted $-L_{0}^{3} \Delta f_{e x}$ as a function of $-t\left[L_{0} / \xi_{0}\right]^{1 / \nu}$, where we have used $\nu=0.6717$ and $\xi_{0}=0.26362$, eq. (6). We find that throughout the function assumes a negative value. In all cases it has a single minimum at $t\left[L_{0} / \xi_{0}\right]^{1 / \nu} \approx$ -5 . The position of the minimum $\beta_{\min }\left(L_{0}\right)$ can be easily determined: It is given by the zero of $\Delta E_{e x}$. We have computed $\beta_{\min }\left(L_{0}\right)$ by linearly fitting $\Delta E_{e x}$ in the neighbourhood of the minimum. In addition to $L_{0}=8.5,16.5$ and 32.5 we performed simulations for $L_{0}=6.5,7.5,9.5,12.5$ and 24.5 at a few values of $\beta$ in the neighbourhood of $\beta_{\text {min }}$. Our results are summarized in table 2 .

The curves for $L_{0}=8.5,16.5$ and 32.5 plotted in figure 1 do not fall on top of each other. E.g. both the position and the value of the minimum are quite different for different $L_{0}$. In order to take corrections into account we have replaced $L_{0}$ by $L_{0, \text { eff }}=L_{0}+L_{s}$, where $L_{s}=1.02(7)$ [13]. To this end, in figure 2 we have plotted $-L_{0, e f f}^{3} \Delta f_{e x}$ as a function of $-t\left[L_{0, e f f} / \xi_{0}\right]^{1 / \nu}$, where we have used the central value of the shift $L_{s}=1.02$. Now indeed the distance between the curves for different $L_{0}$ is much reduced compared with figure 1. The results for $L_{0}=16.5$ and $L_{0}=32.5$ are almost consistent within error bars. Note that using $L_{s}=0.95$ the matching of the data for different $L_{0}$ seems to be better than for $L_{s}=1.02$.

Let us discuss in more detail the results obtained for the minimum of the finite size scaling function $\theta(x)$. Using the numbers given in the third column of table 2 and $L_{s}=1.02$ we get $-\Delta f_{\text {ex,min }} L_{0, \text { eff }}^{3}=-1.365(3),-1.341(6)$ and $-1.311(19)$ for $L_{0}=8.5,16.5$ and 32.5 , respectively. Using instead $L_{s}=0.95$ we get $-1.335(3)$, $-1.325(6)$ and $-1.302(19)$ for $L_{0}=8.5,16.5$ and 32.5, respectively. As our final result we take the one obtained from $L_{0}=32.5$ and $L_{s}=1.02$ :

$$
\theta_{\min }=-1.31(3)
$$

where the error that is quoted takes into account the statistical error and the uncertainty of $L_{s}$.

Next we have fitted our results for $\beta_{\min }$ with the ansatz

$$
t_{\text {min }}\left(1+c t_{\text {min }}\right)\left(L_{0, e f f} / \xi_{0}\right)^{1 / \nu}=x_{\text {min }}
$$




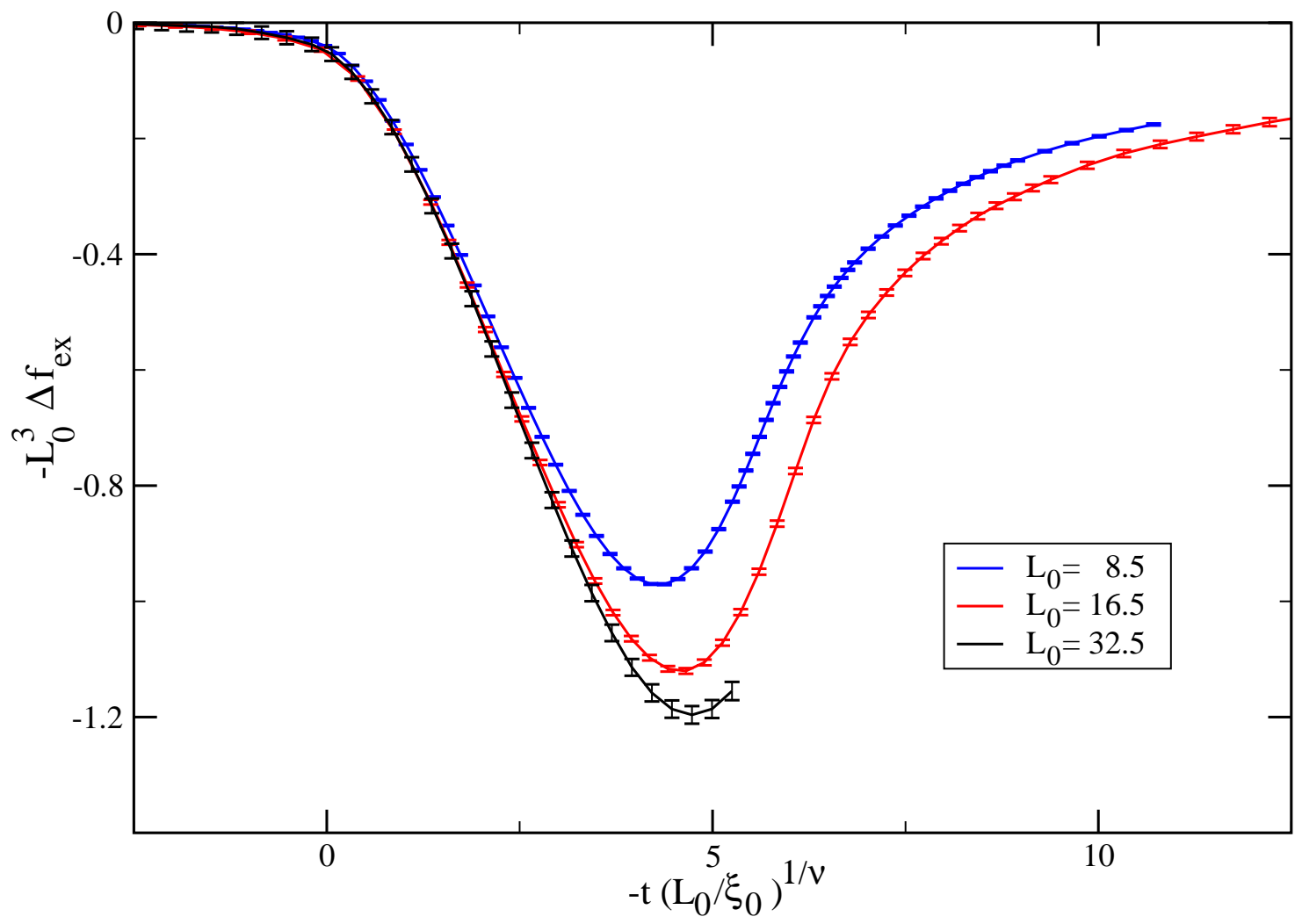

Figure 1: We plot $-L_{0}^{3} \Delta f_{\text {ex }}$ as a function of $-t\left(L_{0} / \xi_{0}\right)^{1 / \nu}$ for $L_{0}=8.5,16.5$ and 32.5 , where we use $\nu=0.6717$ and $\xi_{0}=0.26362$. For a discussion see the text. 


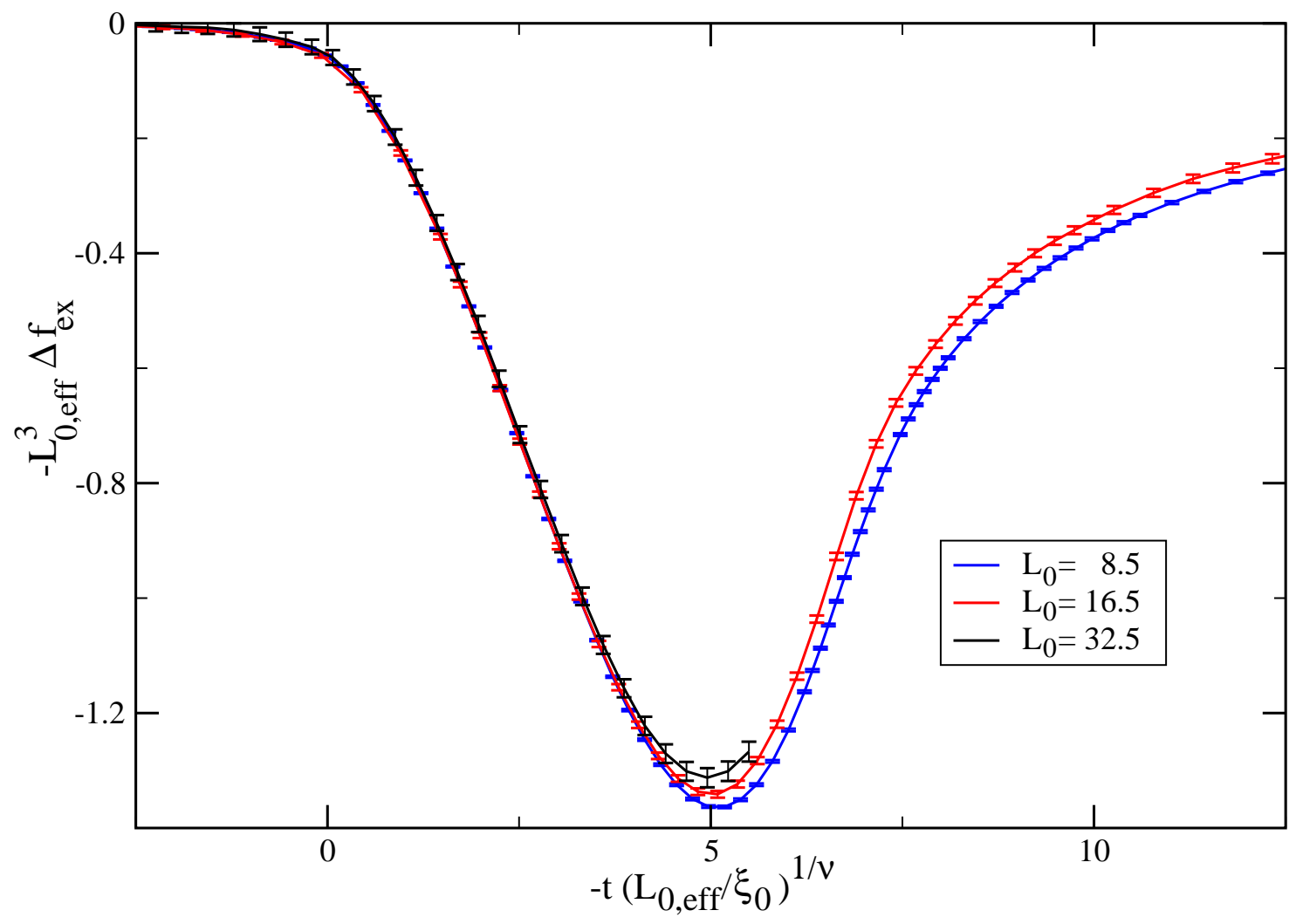

Figure 2: We plot $-L_{0, e f f}^{3} \Delta f_{e x}$ as a function of $-t\left(L_{0, e f f} / \xi_{0}\right)^{1 / \nu}$ for $L_{0}=8.5$, 16.5 and 32.5, where we use $\nu=0.6717, \xi_{0}=0.26362$ and $L_{0, \text { eff }}=L_{0}+L_{s}$ with $L_{s}=1.02$. For a discussion see the text. 
Table 2: We give the position $\beta_{\text {min }}$ of the minimum of the Casimir force and its value $-\Delta f_{\text {ex,min }}$ as a function of the thickness $L_{0}$.

\begin{tabular}{|r|c|c|}
\hline \multicolumn{1}{|c|}{$L_{0}$} & $\beta_{\min }$ & $-\Delta f_{\text {ex } \min }$ \\
\hline 6.5 & $0.54432(2)$ & \\
7.5 & $0.53814(2)$ & \\
8.5 & $0.53354(2)$ & $-0.001582(3)$ \\
9.5 & $0.53010(2)$ & \\
12.5 & $0.52348(2)$ & \\
16.5 & $0.51886(2)$ & $-0.0002494(11)$ \\
24.5 & $0.51463(2)$ & \\
32.5 & $0.51279(2)$ & $-0.0000348(5)$ \\
\hline
\end{tabular}

Table 3: We fit our results for $\beta_{\text {min }}$ with ansatz (20). $L_{0, \text { min }}$ is the smallest thickness of the film that is included into the fit.

\begin{tabular}{|c|l|l|c|}
\hline$L_{0, \min }$ & \multicolumn{1}{|c|}{$x_{\min }$} & \multicolumn{1}{c|}{$c$} & $\chi^{2} /$ d.o.f. \\
\hline 6.5 & $-4.942(6)$ & $1.20(4)$ & 1.47 \\
7.5 & $-4.945(8)$ & $1.17(7)$ & 1.72 \\
8.5 & $-4.956(10)$ & $1.04(10)$ & 1.32 \\
9.5 & $-4.952(12)$ & $1.10(12)$ & 1.64 \\
\hline
\end{tabular}

where $t_{\text {min }}=\beta_{c}-\beta_{\text {min }}$. We have used $\nu=0.6717, \xi_{0}=0.26362, \beta_{c}=0.5091503$ and $L_{s}=1.02$ as input and $c$ and $x_{\min }$ as parameters of the fit. The term $(1+$ $\left.c t_{\text {min }}\right)$ parametrizes analytic corrections. We did not include a correction with the exponent $\theta^{\prime} \approx 1.2$ [35], since within the accuracy of our data they can not be discriminated from the leading analytic correction. The results of these fits are summarized in table 3. The $\chi^{2} /$ d.o.f. is reasonably small starting from $L_{0, \min }=6.5$, where all thicknesses $L_{0}$ with $L_{0} \geq L_{0, \min }$ are included into the fit. Also the estimates for $x_{\text {min }}$ and $c$ do not change much as $L_{0, \min }$ is changed. In order to check the dependence of the results on the value of $L_{s}$ we have repeated the fit for $L_{0, \min }=8.5 \mathrm{using} L_{s}=0.95$ instead of $L_{0}=1.02$. We get the $x_{\min }=-4.943(10)$, $c=0.70(10)$ with $\chi^{2} /$ d.o.f. $=1.12$. As our final result we take

$$
x_{\min }=-4.95(3)
$$


where the error bar covers the statistical error and the uncertainty of $L_{s}$.

Our result for $x_{\min }$ can be compared with those given in the literature. The experimental works [16] give $x_{\min }=-5.45(12)$ (no final result for $\theta_{\min }$ is quoted) and [17] $x_{\min }=-5.7(5)$ and $\theta_{\min }=-1.30(3)$. In [16] and [17] the convention $x=t L_{0}^{1 / \nu}$ is used. In order to convert to $x=t\left[L_{0} / \xi_{0}\right]^{1 / \nu}$ we have used $\xi_{0}=1.422 \AA$ for ${ }^{4} \mathrm{He}$ at vapour pressure as discussed in section 4.2 of [14].

In his Monte Carlo study of the XY model on the simple cubic lattice, Hucht [22] finds $x=-5.3(1)$ and $\theta_{\min }=-1.35(3)$. The authors of [23] have used two different ansätze for the corrections to scaling. Using the first one, they arrive at $x_{\min }=-5.43(2)$ and $\theta_{\min }=-1.396(6)$ and using the second one at $x_{\min }=-5.43(2)$ and $\theta_{\min }=-1.260(5)$. Note that the ansätze used by [23], see eqs. $(20,21,22,23)$ of [23], provide only an overall $x$-independent factor; therefore they do not allow for any correction to scaling of $x_{\min }$.

Our results for $\theta_{\min }$ is in good agreement with both the experiment [17] as well as with previous Monte Carlo studies of the XY model [22, 23]. On the other hand, the position of the minimum $x_{\text {min }}$ differs by several times the quoted error bar from both the experiment [16, 17] as well as from Monte Carlo studies of the XY model [22, 23].

Finally, in figure 3 we take into account the analytic corrections that we have detected fitting the position $t_{\text {min }}$ of the minimum of the Casimir force. To this end, we have replaced the argument $t\left(L_{0, \text { eff }} / \xi_{0}\right)^{1 / \nu}$ by $t(1+1.04 t)\left(L_{0, \text { eff }} / \xi_{0}\right)^{1 / \nu}$. The coefficient of the analytic correction is taken from the fit where we have fixed $L_{s}=1.02$ and $L_{0, \min }=8.5$. Now we find an almost perfect match between the curves obtained from $L_{0}=8.5,16.5$ and 32.5 .

\subsection{Comparison with other theoretical approaches}

Krech and Dietrich [18, 19] have computed the finite size scaling function $\theta$ in the high temperature phase using the $\epsilon$-expansion up to $\mathrm{O}(\epsilon)$. In figure 4 we plot their result for the XY universality class $(N=2)$ setting $\epsilon=1$. For comparison we plot our results for $L_{0}=8.5, L_{0}=16.5$ and $L_{0}=32.5$. We have taken into account leading boundary corrections by replacing $L_{0}$ by $L_{0, \text { eff }}=L_{0}+L_{s}$, where we have taken $L_{s}=1.02$.

Comparing with the $\epsilon$-expansion we can estimate the systematical error caused by setting $\Delta f_{e x}\left(\beta_{0}\right)=0$ in eq. (17): We read off from the $\epsilon$-expansion that $\theta \approx$ $-0.0035,-0.0022,-0.0015$ for our choices of $\beta_{0}$ for $L_{0}=8.5,16.5$ and 32.5. Taking into account this error, we see a good agreement of our Monte Carlo results and the

$\epsilon$-expansion down to $L_{0} / \xi \approx 1$. The curve obtained from the $\epsilon$-expansion flattens 


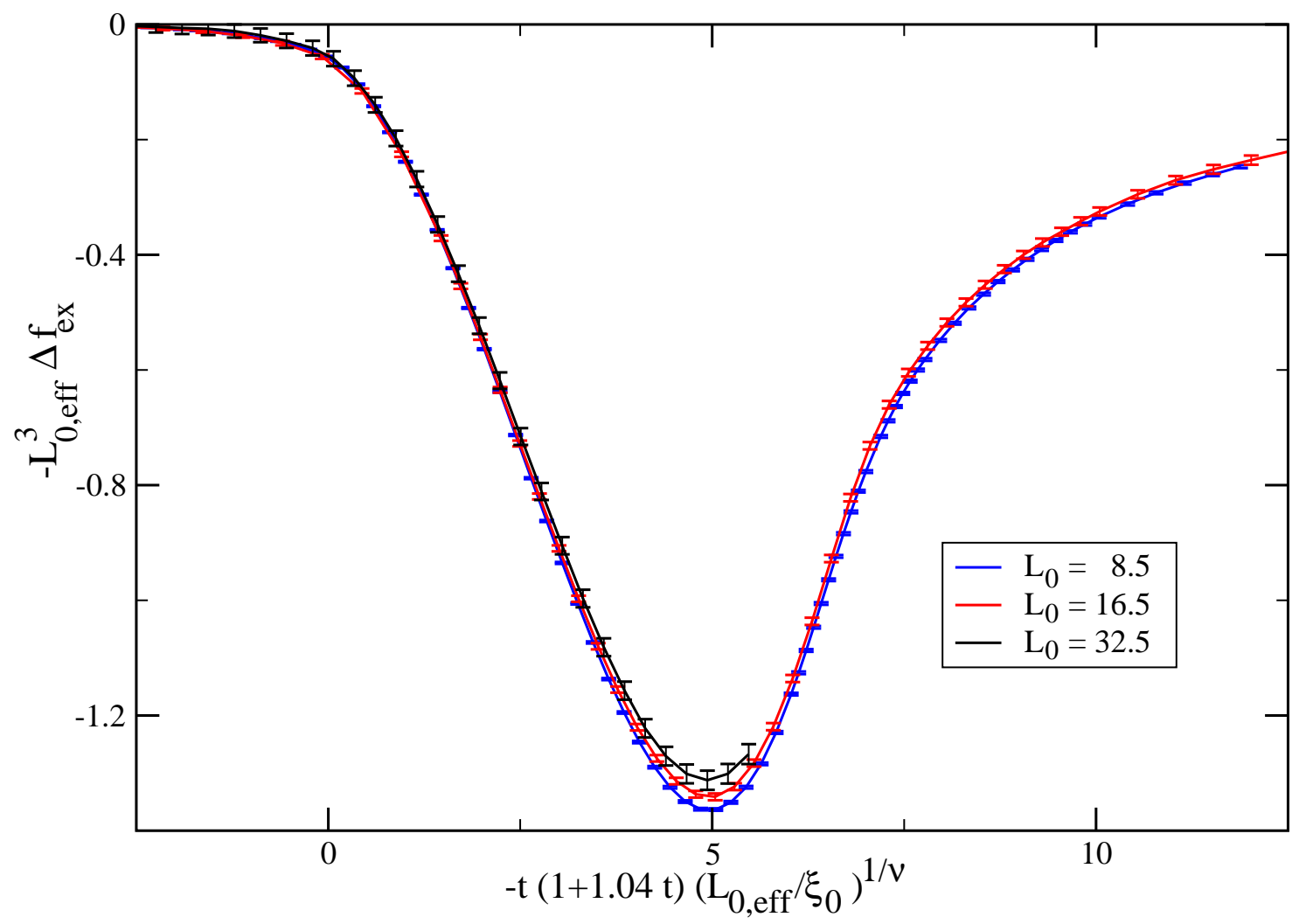

Figure 3: We plot $-L_{0, e f f}^{3} \Delta f_{e x}$ is plotted as a function of $-t(1+1.04 t)\left(L_{0, e f f} / \xi_{0}\right)^{1 / \nu}$ for $L_{0}=8.5,16.5$ and 32.5, where we use $\nu=0.6717, \xi_{0}=0.26362$ and $L_{0, e f f}=$ $L_{0}+L_{s}$ with $L_{s}=1.02$. For a discussion see the text. 
as the critical point is approached. At the critical point the slope vanishes. In contrast, in our case, the curve steepens as the critical point is approached.

The authors of [20] have computed the finite size scaling function $\theta$ using a renormalized mean field approach. While this approach correctly reproduces qualitative features of the finite size scaling function $\theta$ it fails to give quantitatively accurate results. In particular the authors of [20] find $x_{\min }=-\pi^{2} \approx-9.8696$ and $\theta_{\min } \approx-6.92$. I.e. The position of the minimum is overestimated by about a factor of 2 and its value by a factor of about 5 .

The authors of [36] incorporate fluctuation effects into their mean-field analysis. Qualitatively they get the finite size scaling function $\theta$ for the whole range of $x$ right. They adjust the two parameters of their solution for the low temperature phase (eq. (17) of [36] ) such that the minimum of $\theta$ found in the experiments [16, 17] is reproduced. We did the same exercise, adjusting to our result for the minimum of $\theta$. We find no good match between $\theta$ computed in [36] and ours. Our minimum is much more peaked than that of [36].

\subsection{Comparison with experimental results}

Finally we compare our result for the finite size scaling function $\theta$ with experiments [16, 17]. In [16] films of thicknesses between 298 and $588 \AA$ have been studied. In figure 5 we have plotted the data obtained from capacitor 1 which corresponds to the thickness $575 \AA$ of the film at temperatures $T>T_{\lambda}$. This set of data is the smoothest among the five sets given in [16, 37]. The results of [17] are, in the range of temperatures we are interested in, less precise than those of [16]. In the tables provided by the authors [37] the finite size scaling function $\theta$ is given as a

function of $\left(T / T_{\lambda}-1\right) L_{0}^{1 / \nu}$. In order to compare with our results we have converted this to $\left(T / T_{\lambda}-1\right)\left(L_{0} / \xi_{0}\right)^{1 / \nu}$, using $\xi_{0}=1.422 \AA$. For comparison we give our result obtained from $L_{0}=16.5$, where we have taken into account the boundary correction by replacing $L_{0}$ by $L_{0, \text { eff }}$ and the leading analytic correction as discussed above. Furthermore, we give the asymptotic value [38, 39]

$$
\lim _{x \rightarrow \infty} \theta(x)=-\frac{\zeta(3)}{8 \pi} \approx-0.04783
$$

obtained from the spin wave approximation.

As already observed by the authors of [22, 23] there is a qualitative agreement among the result obtained from Monte Carlo simulations of lattice models and the experiment. There is a reasonable agreement of the position of the minimum $x_{\text {min }}$, as already discussed above. For $x<x_{\min }$ our result is in good agreement with 


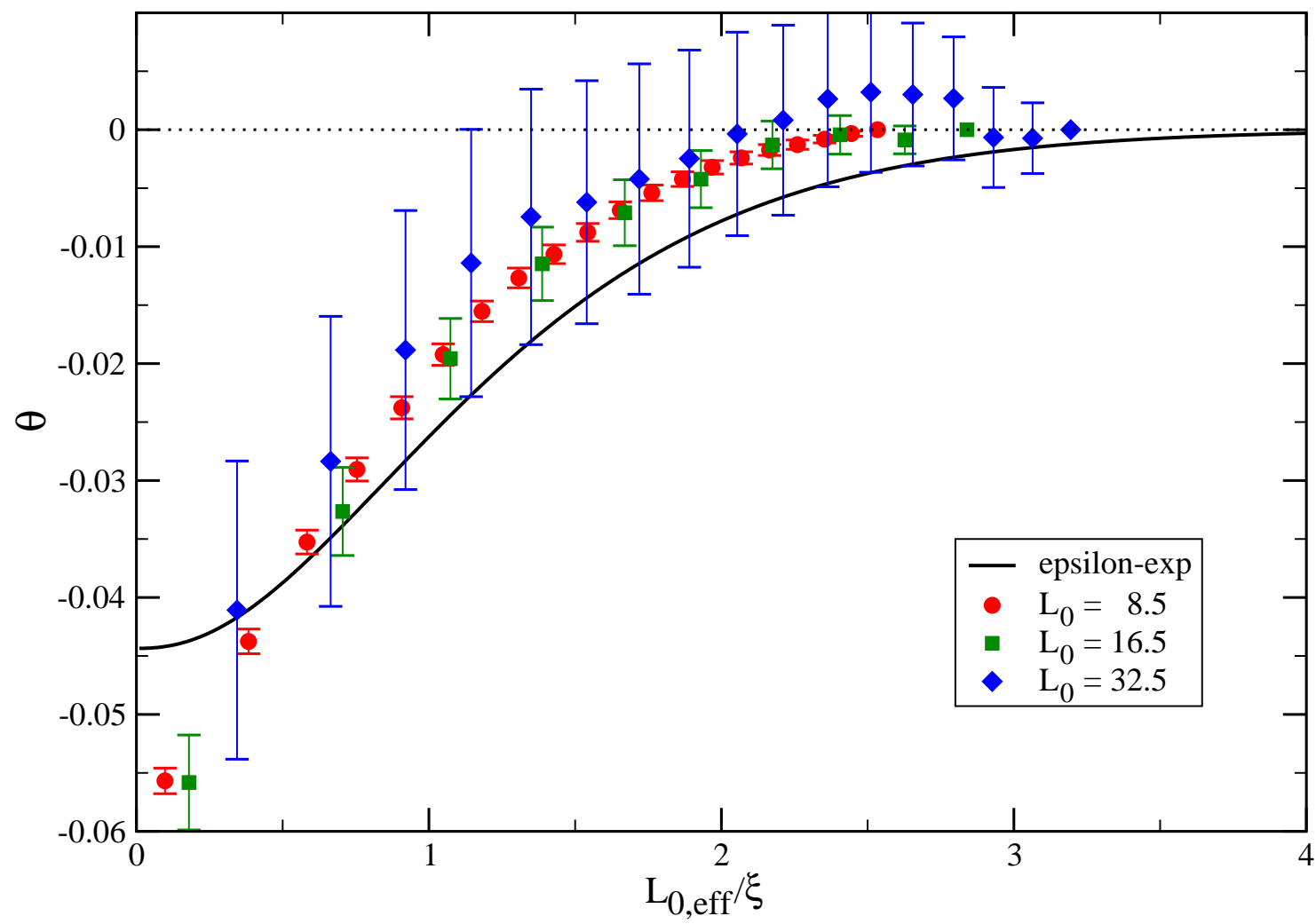

Figure 4: We plot the finite size scaling function $\theta$ as a function of $L_{0} / \xi$ in the high temperature phase obtained by Krech and Dietrich [18, 19] using the $\epsilon$-expansion. For comparison we give our results obtained for $L_{0}=8.5, L_{0}=16.5$ and $L_{0}=32.5$. In the case of our data the leading boundary correction is taken into account by replacing $L_{0}$ by $L_{0, e f f}=L_{0}+L_{s}$ with $L_{s}=1.02$. For a discussion see the text. 
that of the experiment. In contrast for $x>x_{\text {min }}$ the experimental value of $\theta$ is clearly smaller than ours. For $x \approx-30$ the experimental result (capacitor 1 of [16]) assumes $\approx-0.19$ and is decreasing again for smaller values of $x$. This is clearly different from the prediction (22) of the spin wave approximation.

In ref. [39] it was argued that this discrepancy could be explained by fluctuations of the surface resulting in

$$
\lim _{x \rightarrow-\infty} \tilde{\theta}(x)=-\frac{11 \zeta(3)}{32 \pi} \approx-0.1315
$$

This goes indeed in the right direction, can however not fully explain the difference between the experimental result and the theoretical prediction (22).

\section{Summary and Conclusions}

We have simulated the improved two component $\phi^{4}$ model on the simple cubic lattice. This model shares with the $\lambda$-transition of ${ }^{4} \mathrm{He}$ the three dimensional XY universality class. We consider the thin film geometry. In order to mimic the vanishing order parameter at the surface of ${ }^{4} \mathrm{He}$ films near the $\lambda$-transition, we impose Dirichlet boundary conditions with a vanishing field.

Restricting the system to a finite geometry leads to an effective force called thermodynamic Casimir force. Its behaviour in the neighbourhood of the critical point is characterized by a universal finite size scaling function. We have computed this function and have compared our result with that obtained from experiments on films of ${ }^{4} \mathrm{He}$ [16, 17, 37], previous Monte Carlo simulations of the XY model on the simple cubic lattice [21, 22, 23], field theoretic methods [18, 19] and mean field approaches [20, 36].

The thermodynamic Casimir force is given as minus the derivative of the excess free energy of the film with respect to its thickness $L_{0}$. On the lattice, this is approximated by the finite difference of films of the thickness $L_{0}+1 / 2$ and $L_{0}-1 / 2$, where $L_{0}+1 / 2$ is integer.

In general it is impossible to compute free energies from a single Monte Carlo simulation. To circumvent this problem, divide and conquer strategies are employed. Different strategies have been proposed in [21, 23] and [22]. Here we essentially follow [22]: We compute the derivative of the excess energy with respect to $L_{0}$ for a dense grid of temperature values in the neighbourhood of the critical point. The corresponding result for the free energy is then obtained by numerical integration. 


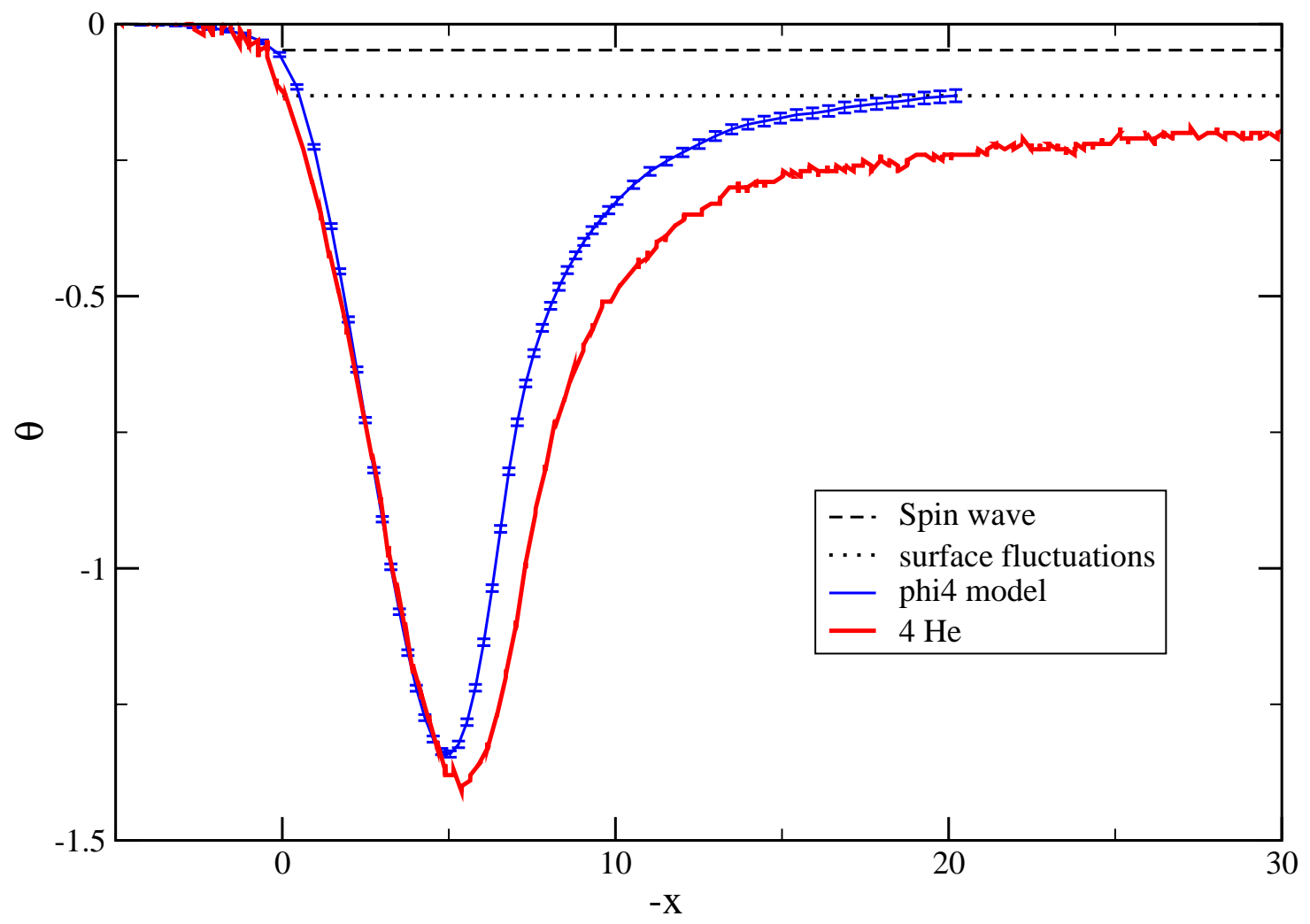

Figure 5: We plot the finite size scaling function $\theta(x)$ obtained from an experiment on a thin film of ${ }^{4} \mathrm{He}$ [16, 37], where $x=\left(T / T_{\lambda}-1\right)\left(L_{0} / \xi_{0}\right)^{1 / \nu}$ with $\xi_{0}=1.422 \AA$. For comparison we give our result obtained from $L_{0}=16.5$, where we have taken into account boundary and analytic corrections as in figure 3. Furthermore we give the asymptotic value -0.04783 for $x \rightarrow-\infty$ (dashed line). The authors of [20] have argued that fluctuations of the surface of the ${ }^{4} \mathrm{He}$ film gives an additional contribution to the Casimir force. The corresponding asymptotic value -0.1315 is given by the dotted line. For a discussion see the text. 
As discussed in ref. [23], corrections to scaling are numerically quite large for the thicknesses that can be studied at present. The main purpose of the present work is to get better control over these corrections as in previous work [22, 23].

To this end, we have studied the $\phi^{4}$ model at $\lambda=2.1$. In this model leading corrections to scaling (and finite size scaling) which are $\propto L_{0}^{-\omega}$, with $\omega=0.785(20)$, are suppressed at least by a factor of 20 compared with the XY model [24].

Boundary effects lead to corrections $\propto L_{0}^{-1}$. These corrections can be cast into the form $L_{0, \text { eff }}=L_{0}+L_{s}$. In [13] we have determined $L_{s}=1.02(7)$ for the two component $\phi^{4}$ model at $\lambda=2.1$ by using a finite size scaling study at the critical point of the three dimensional system. We have verified that this choice of $L_{s}$ indeed eliminates the leading boundary correction in the scaling of the temperature of the Kosterlitz-Thouless transition [13] and the specific heat of thin films [14]. Also here we confirm that corrections can be essentially eliminated by replacing $L_{0}$ by $L_{0, e f f}=L_{0}+L_{s}$ with $L_{s}=1.02(7)$. Remaining discrepancies can be fitted by analytic corrections.

Essentially we confirm the results obtained by previous Monte Carlo simulations of the three dimensional XY model [22, 23] for the finite size scaling function $\theta$. The main discrepancy with these previous works is the position of the minimum $x_{\min }=-4.95(3)$ compared with $x_{\min }=-5.3(1)$ 22] and $x_{\min }=-5.43(2)$ [23].

We should note that the Monte Carlo simulation of lattice models is at the moment the only theoretical method that allows for a quantitatively accurate calculation of $\theta$ in the low temperature phase not too far from the critical point. The $\epsilon$-expansion gives correctly the behaviour in the high temperature phase. The spin wave approximation gives the exact result in the limit $x \rightarrow-\infty$. The mean field calculation of [20] reproduces only qualitatively the features of the scaling function. Quantitatively it is not satisfactory: the position of the minimum is wrongly estimated by a factor of almost 2 and its value by a factor of about 5 .

Qualitatively the Monte Carlo studies of lattice models nicely reproduce the finite size scaling function obtain from the experimental data [16, 17, 37] for films of ${ }^{4} \mathrm{He}$. For $x>x_{\min }$ there is a very good quantitative agreement between the two. In contrast, for $x<x_{\min }$ the value obtained from the experiment is clearly smaller than that of the Monte Carlo studies. At large values of $-x$ the spin wave approximation should become exact. Also in this regime, the experiments produce numbers that are too small compared with the theoretical one. The authors of [20] explain this discrepancy by fluctuations of the surface of the ${ }^{4} \mathrm{He}$ film. Their result indeed reduces but not completely eliminates the difference between experiment and theory. Therefore it might be interesting to perform Monte Carlo simulations of a lattice model that incorporates fluctuations of the surface of the film. 


\section{Acknowledgements}

This work was supported by the DFG under the grant No HA 3150/2-1.

\section{References}

[1] Fisher M E and de Gennes P-G, Phenomena at the walls in a critical binary mixture, 1978 CR Acad. Sci. Paris B 287207

[2] Wilson K G and Kogut J, The renormalization group and the $\epsilon$-expansion, 1974 Phys. Rep. C 1275

[3] Fisher M E, The renormalization group in the theory of critical behavior, 1974 Rev. Mod. Phys. 46597

[4] Fisher M E, Renormalization group theory: Its basis and formulation in statistical physics, 1998 Rev. Mod. Phys. 70653

[5] Pelissetto A and Vicari E, Critical Phenomena and Renormalization-Group Theory, 2002 Phys. Rept. 368549 [arXiv:cond-mat/0012164]

[6] Barmatz M, Hahn I, Lipa J A, and Duncan R V, Critical phenomena in microgravity: Past, present, and future, 2007 Rev. Mod. Phys. 791

[7] M. N. Barber Finite-size Scaling in Phase Transitions and Critical Phenomena, Vol. 8, eds. C. Domb and J. L. Lebowitz, (Academic Press, 1983)

[8] Finite Size Scaling and Numerical Simulation of Statistical Systems, ed. V. Privman, (World Scientific, 1990).

[9] Gasparini F M, Kimball M O, Mooney K P, and Diaz-Avila M, Finite-size scaling of ${ }^{4} \mathrm{He}$ at the superfluid transition, 2008 Rev. Mod. Phys. 801009

[10] Kosterlitz J M and Thouless D J, Ordering, metastability and phase transitions in two-dimensional systems 1973 J. Phys. C 6 1181; Kosterlitz J M, The critical properties of the two-dimensional XY model, 1974 J. Phys. C 71046

[11] José J V, Kadanoff L P, Kirkpatrick S and Nelson D R, Renormalization, vortices, and symmetry-breaking perturbations in the two-dimensional planar model, 1977 Phys. Rev. B 161217 
[12] Amit D J, Goldschmidt Y Y and Grinstein G, Renormalisation group analysis of the phase transition in the $2 D$ Coulomb gas, Sine-Gordon theory and XY model, 1980 J. Phys. A 13585

[13] Hasenbusch M, Kosterlitz-Thouless transition in thin films: A Monte Carlo study of three-dimensional lattice models, 2009 J. Stat. Mech. P02005 arXiv:0811.2178,

[14] Hasenbusch M, The specific heat of thin films near the $\lambda$-transition: A Monte Carlo study of an improved three-dimensional lattice model, 2009 arXiv:0904.1535]

[15] J.G. Brankov, D.M. Dantchev, and N.S. Tonchev, Theory of Critical Phenomena in Finite-Size Systems - Scaling and Quantum Effects (World Scientific, Singapore, 2000).

[16] Garcia R and Chan M H W, Critical Fluctuation-Induced Thinning of ${ }^{4} \mathrm{He}$ Films near the Superfluid Transition, 1999 Phys. Rev. Lett. 831187

[17] Ganshin A, Scheidemantel S, Garcia R, and Chan M H W, Critical Casimir Force in ${ }^{4}$ He Films: Confirmation of Finite-Size Scaling, 2006 Phys. Rev. Lett. 97075301

[18] Krech M and Dietrich S, Free energy and specific heat of critical films and surfaces, 1992 Phys. Rev. A 461886

[19] Krech M and Dietrich S, Specific heat of critical films, the Casimir force and wetting films near end points, 1992 Phys. Rev. A 461922

[20] Zandi R, Shackell A, Rudnick J, Kardar M and Chayes L, Thinning of superfluid films below the critical point, 2007 Phys. Rev. E 76 (2007) 030601 cond-mat/0703262

[21] Vasilyev O, Gambassi A, Maciolek A, and Dietrich S, Monte Carlo simulation results for critical Casimir forces, 2007 Europhys. Lett. 8060009 arXiv:0708.2902

[22] Hucht A, Thermodynamic Casimir Effect in ${ }^{4} H e$ Films near $T_{c}$ : Monte Carlo Results, 2007 Phys. Rev. Lett. 99185301 [arXiv:0706.3458] 
[23] Vasilyev O, Gambassi A, Maciolek A, and Dietrich S, Universal scaling functions of critical Casimir forces obtained by Monte Carlo simulations, 2008 arXiv:0812.0750

[24] Campostrini M, Hasenbusch M, Pelissetto A, and Vicari E, Theoretical estimates of the critical exponents of the superfluid transition in He4 by lattice methods, 2006 Phys. Rev. B 74144506 [cond-mat/0605083

[25] Diehl H W, Dietrich S, and Eisenriegler E, Universality, irrelevant surface operators, and corrections to scaling in systems with free surfaces and defect planes, 1983 Phys. Rev. B 272937

[26] Capehart T W and Fisher M E, Susceptibility scaling functions for ferromagnetic Ising films, 1976 Phys. Rev. B 135021

[27] Hasenbusch M and Török T, High precision Monte Carlo study of the 3D XY-universality class, 1999 J. Phys. A 326361 cond-mat/9904408

[28] Campostrini M, Hasenbusch M, Pelissetto A, Rossi P, and Vicari E, Critical behavior of the three-dimensional XY universality class, 2001 Phys. Rev. B 63 214503 cond-mat/0010360]

[29] Hasenbusch M, The three-dimensional XY universality class: A high precision Monte Carlo estimate of the universal amplitude ratio $A_{+} / A_{-}, 2006 \mathrm{~J}$. Stat. Mech. P08019 cond-mat/0607189]

[30] Hasenbusch M, A Monte Carlo study of the three-dimensional XY universality class: Universal amplitude ratios, J. Stat. Mech. (2008) P12006 arXiv:0810.2716

[31] Lipa J A, Nissen J A, Stricker D A, Swanson D R and Chui T C P, Specific heat of liquid helium in zero gravity very near the $\lambda$-point, 2003 Phys. Rev. B 68174518 arXiv:cond-mat/0310163

[32] Wolff U, Collective Monte Carlo Updating for Spin Systems, 1989 Phys. Rev. Lett. 62361

[33] Hasenbusch M, Pinn K and Vinti S, Critical Exponents of the 3D Ising Universality Class From Finite Size Scaling With Standard and Improved Actions, 1999 Phys. Rev. B 5911471 hep-lat/9806012 
[34] Saito M, An Application of Finite Field: Design and Implementation of 128-bit Instruction-Based Fast Pseudorandom Number Generator, PhD thesis, Dept. of Math., Graduate School of Science, Hiroshima University, Advisor: M. Matsumoto; The numerical program and a detailed description can be found at "http://www.math.sci.hiroshima-u.ac.jp/ ^m-mat/MT/SFMT/index.htmll"

[35] Newman K E and Riedel E K, Critical exponents by the scaling-field method: The isotropic N-vector model in three dimensions, 1984 Phys. Rev. B 306615

[36] Biswas S, Bhattacharjee J K, Samanta H S, Bhattacharyya S, and Hu B, Theory of the critical Casimir force for He-4 film arXiv:0808.0390]

[37] http://users.wpi.edu/ garcia/casimirdata/

[38] Li H and Kardar M, Fluctuation-Induced Forces between Rough Surfaces, 1991 Phys. Rev. Lett. 673275

[39] Zandi R, Rudnick J and Kardar M, Casimir Forces, Surface Fluctuations, and Thinning of Superfluid Film, 2004 Phys. Rev. Lett. 93155302 